\title{
Rationale, Design, and Methodology of a Prospective Cohort Study for Coping with Behavioral and Psychological Symptoms of Dementia: The RECage Project
}

Eleni Poptsi, Magda Tsolaki, Sverre Bergh, Bruno Mario Cesana, Alfonso Ciceone, Andrea Fabbo, Giovanni B. Frisoni, Lutz Frölich, Anna Giulia Guazzarini, Jacques Hugon, Sara Fascendini, Sara Lavolpe, Patrizia Mecocci, Oliver Peters and Carlo Alberto Defanti

[Journal of Alzheimer's Disease, 80(4) 2021, 1613-1627, DOI 10.3233/JAD-201215]

https://content.iospress.com/articles/journal-of-alzheimers-disease/jad201215.

When this paper was originally published two author names, Patrizia Mecocci and Oliver Peters, were unintentionally omitted from author list. The correct list of authors and affiliations is:

Eleni Poptsi ${ }^{\mathrm{a}, \mathrm{b}}$, Magda Tsolakib,c, Sverre Bergh ${ }^{\mathrm{d}}$, Bruno Mario Cesana ${ }^{\mathrm{e}}$, Alfonso Ciceone $^{\mathrm{f}}$, Andrea Fabbo $^{\mathrm{g}}$, Giovanni B. Frisoni ${ }^{\mathrm{h}}$, Lutz Frölich ${ }^{\mathrm{i}}$, Anna Giulia Guazzarini ${ }^{\mathrm{j}}$, Jacques Hugon ${ }^{\mathrm{k}}$, Sara Fascendini ${ }^{1}$, Sara Lavolpe ${ }^{\mathrm{m}}$, Patrizia Mecocci ${ }^{\mathrm{j}}$, Oliver Peters ${ }^{\mathrm{n}, \mathrm{o}}$, and Carlo Alberto Defanti ${ }^{1}$

${ }^{a}$ Laboratory of Psychology, Section of Cognitive and Experimental Psychology, Faculty of Philosophy, School of Psychology, Aristotle University of Thessaloniki (AUTh), Macedonia, Greece ${ }^{b}$ Greek Association of Alzheimer's Disease and Related Disorders (GAADRD), Thessaloniki, Macedonia, Greece

${ }^{c} 1$ st Department of Neurology, School of Medicine, Aristotle University of Thessaloniki (AUTh), Macedonia, Greece

${ }^{d}$ Research Centre for Age-Related Functional Decline and Disease, Innlandet Hospital Trust, Ottestad, Norway

${ }^{e}$ Department of Clinical Sciences and Community Health, Unit of Medical Statistics, Biometry and Bioinformatics “Giulio A. Maccacaro”, Faculty of Medicine and Surgery, University of Milan, Milan, Italy

${ }^{f}$ Department of Neurology with Neurosurgical Activity, "Carlo Poma" Hospital, ASST di Mantova, Manua, Italy

${ }^{g}$ Direttore UOC di Geriatria-Disturbi Cognitivi e Demenze, Dipartimento Cure Primarie AUSL, Modena, Italy

${ }^{h}$ Memory Clinic, University Hospitals and University of Geneva, Geneva, Switzerland

${ }^{i}$ Department of Geriatric Psychiatry, Central Institute of Mental Health, Medical Faculty Mannheim, Heidelberg University, Mannheim, Germany 
${ }^{j}$ Institute of Gerontology and Geriatrics, Department of Medicine and Surgery, University of Perugia, Perugia, Italy

${ }^{k}$ Center of Cognitive Neurology, Université de Paris, Paris, France

${ }^{l}$ FERB Alzheimer Centre, Gazzaniga, Italy

${ }^{m}$ Department of Neurology and CDCD Centre Humanitas Gavazzeni, Bergamo, Italy

${ }^{n}$ Charité-Universitätsmedizin Berlin, Campus Benjamin Franklin, Department of Psychiatry, Berlin, Germany

${ }^{o}$ German Center for Neurodegenerative Disease (DZNE), Berlin, Germany

The PDF and HTML versions of the original publication have been updated to reflect this change. 